\title{
Applications of Medical Devices in Healthcare Industry
}

\author{
Chandan B.V. ${ }^{1}$, Balamuralidhara V. ${ }^{2}$, Gowrav M.P. ${ }^{3}$, Vishakharaju Motupalli ${ }^{4}$ \\ 1. 2. 3,4 Pharmaceutical Regulatory Affairs Group, Department of Pharmaceutics, JSS College of Pharmacy, \\ Mysuru. JSS Academy of Higher Education and Research, Mysuru, Karnataka, India.
}

\begin{abstract}
In the health-care industry, use of medical devices is becoming increasingly significant. There are currently over 8000 generic medical device categories, with some containing pharmaceutical active ingredients. The potential growth of the medical devices in the healthcare industry helps the healthcare system stupendously in diagnosis, treatment, pathogen tracking, patient monitoring and many more aspects of serving the human race in healthcare terms. Medical device utilization is becoming an increasingly crucial part of a healthcare professional's job. Personal users of medical devices must be taught and educated regularly to guarantee that they are proficient in the usage of equipment.

Medical gadgets are becoming increasingly important in the health-care market. Keeping up with regulatory regulations and incorporating them into the process is one of the most difficult elements of creating and manufacturing medical devices. Tighter regulatory systems are needed to ensure that products entering the market are both safe and effective. Keeping up with regulatory regulations and incorporating them into the process is one of the most difficult elements of creating and manufacturing medical devices. A company that fails to succeed in this endeavour could lose thousands of dollars due to the amount of time it takes to do it.
\end{abstract}

\section{KEY WORDS}

Medical Device, Artificial Intelligence, Machine Learning, Design and Development, Diagnosis, Disease Management.
Corresponding Author:

Dr. Balamuralidhara $V$., Associate Professor,

Pharmaceutical Regulatory Affairs Group, Department of Pharmaceutics,

JSS College of Pharmacy, Mysuru.

JSS Academy of Higher Education and Research, Mysuru, Karnataka, India - 570015

E-mail: baligowda@hotmail.com

DOI: $10.14260 /$ jemds/2021/692

How to Cite This Article:

Chandan BV, Balamuralidhara V, Gowrav $M P$, et al. Applications of medical devices in healthcare industry. J Evolution Med Dent Sci 2021; 10(38):3419-3423, DOI: 10.14260/jemds/2021/692

Submission 21-06-2021,

Peer Review 18-08-2021,

Acceptance 25-08-2021,

Published 20-09-2021.

Copyright (C) 2021 Chandan B.V. et al. This is an open access article distributed under Creative Commons Attribution License [Attribution 4.0 International (CC BY 4.0)] 


\section{BACKGROUND}

Medical devices are crucial in the diagnosis and treatment of a wide range of diseases, especially in surgical specialities, radiology, and critical care.4,1 A medical device is an instrument, apparatus, implement, contrivance, implant, invitro reagent, or another related article that is recognised in the National Formulary, United States Pharmacopoeia, or other supplements and is projected to affect the structure or any function of the body of mammals, as well as the diagnosis of disease, as defined by the Food, Drug, and Cosmetic Act. ${ }^{5}$

Medical equipment is necessary for a wide range of health-care services. Rather than being considered services in and of themselves, medical devices are often employed as inputs in the delivery of health care services. ${ }^{5,2}$ The medical device sector is a vital part of the wider health-care system, and it plays a crucial role in developing innovative medical technology that can help doctors diagnose and treat illnesses more effectively. ${ }^{5}$ Medical device firms, whether large or small, play a role in the creation of innovative medical equipment. Small medical device firms are largely focused on the development of novel medical technology, and their work is often strictly concentrated on a single therapeutic area. ${ }^{5}$

Industry Scenario: The medical device industry has the potential to grow at a rate of $28 \%$ per year by 2025 , reaching $\$ 50$ billion. ${ }^{7}$

Medical devices are divided into five categories:

1. Needles and syringes are examples of consumables and disposables.

2. Diagnostic imaging includes MRIs, X-rays, and ultrasounds, among other procedures.

3. Dentures, braces, and other dental products

4. Knee implants and artificial joints are examples of orthopaedics and prosthetics.

5. Hearing aids and pacemakers are examples of patient aids. ${ }^{8}$

The study of archaeology and Roman medical literature has revealed that numerous types of medical equipment were widely used in ancient Rome. Medical devices were regulated in the United States in 1938 under the Federal Food, Drug, and Cosmetic Act (FD \& C Act). The Medical Device Rule (MDR) is a European regulation that has been in effect since 2017.9,8 MNCs dominated the Indian medical equipment business in the 1960 s, with a $90 \%$ market share. The Indian Patent Act of 1970 and the drug price control order gave the government control after 1970, and Indian enterprises began to emerge. After the beginning of the "Make in India" programme in 2014, India's medical device industry has become self-sufficient. ${ }^{9}$

These days the medical device industry produces a wide range of products, from surgical dressings to prosthetic joints to imaging equipment, and is a key player in the development of new medical technology that can aid in the diagnosis and treatment of diseases. ${ }^{10}$

\section{DISCUSSION}

Medical devices are items / products that aren't absorbed or metabolised by the body and are used for "diagnosis, cure, mitigation, therapy, or prevention of a disease."
To assure product safety, medical equipment were made in accordance with preset compliances. Previously, medical device distributors, importers, and manufacturers sold their products without obtaining a licence. Unfortunately, the government suspended such privileges a few years ago in order to preserve the safety of human life. ${ }^{11}$

Medical device development and innovation must continue to improve to ensure the global quality of life. Syringes, wheelchairs, pacemakers, x-ray equipment, orthopaedic devices, coronary artery stents, and other medical devices are all included under the term "medical devices."

Regardless, there is no denying the medical device healthcare industry's critical role in ensuring the health of an increasingly health-conscious populace. It has turned into a boon that diseases can now be discovered sooner, and diagnosis, treatment, and patient monitoring have all improved as a result of medical devices. ${ }^{12}$

Leap forward of medical devices and tempering of medical devices have also been critical in lowering healthcare costs at a time when health services around the world, including the National Health Service, appear to be under more financial strain than ever. ${ }^{12}$

\section{Design and Maturing of Medical Devices throughout the Era}

Medical device design and maturing is a cyclical process that involves constructing a device for a certain purpose or group of duties, then continuously re-evaluating and improving its performance until the device becomes obsolete. ${ }^{13}$

Design and development begin with concept generation and the formulation of a concept that is then developed, engineered, and prototyped if it is determined to be both financially and clinically feasible. Bench testing, which is done by simulating the use of the product, and animal testing, as well as any necessary redesign work, are all part of the preclinical phase. ${ }^{13}$

The planned medical device, as well as the procedure by which it will be created, is scrutinised throughout the process for defects that could jeopardise the device's safety, market viability, regulatory approval, customer satisfaction, usability, or profitability. Any flaws are addressed, and the improvements are incorporated into the final design. Because many current medical equipment have wireless networking capabilities, cybersecurity and interoperability must be factored into the design. Human participants are used in clinical testing to uncover defects and check product strengths. Production and commercialization of a device can commence once the product design and manufacturing process have been ratified and authorised by the US Food and Drug Administration (FDA). ${ }^{13}$

\section{Artificial Intelligence in Medical Devices}

The field of artificial intelligence (AI) is enlarging at a breakneck pace. Global venture funding for AI-focused firms increased by an estimated $50 \%$ between 2017 and 2018, topping $\$ 19$ billion. ${ }^{14}$ Though it's easy to notice AI's impact on the IT industry, the reality is that AI is branching out and impacting other industries as well, including medical devices. According to the authors of a 2020 Nature paper, $64 \mathrm{AI}$ 
medical devices / algorithms have already been cleared or authorised by the FDA. ${ }^{15}$

Any medical device that can collect health data, evaluate it, and make / inform decisions based on the data is referred to as "medical artificial intelligence." 15

Original equipment manufacturers (OEMs) are beginning to move beyond linked devices to offer predictive goods based on AI technologies, with businesses such as GE Healthcare, Siemens Healthineers, and Varian Medical Systems leading the way. Unsurprisingly, providing this level of service necessitates a commitment to a single trader's offering. There is currently limited connectivity betwixt competing original equipment manufacturers' offerings. The winning vendor will almost definitely have a monopoly if one of these systems is purchased. ${ }^{15,16}$

Artificial intelligence (AI) is a combination of science and engineering to create intelligent devices, particularly intelligent computer programmes. Models based on statistical data analysis, expert systems that largely rely on if-then statements, and machine learning are all examples of artificial intelligence techniques. ${ }^{15}$

Machine learning is an artificial intelligence methodology for designing and training software algorithms that can learn from and act on data. Software engineers can employ machine learning to construct a 'locked' algorithm that does not modify its function or an 'adaptive' algorithm that changes its behaviour over time based on new data. ${ }^{15}$

For example:

- An imaging system that employs algorithms to provide diagnostic information for patients with skin cancer is an example of artificial intelligence and machine learning technology in action.

- A smart sensor that predicts the likelihood of a heart attack. $^{15}$

\section{Transforming Medical Devices with Artificial Intelligence and Machine Learning}

Artificial intelligence (AI) and machine learning (ML) technologies have the potential to revolutionize health care by extracting new and essential insights from the massive amounts of data created every day during the delivery of health care. Manufacturers of medical devices are incorporating these technologies into their products to help health care providers and improve patient care. One of the most significant advantages of AI / ML in software is its potential to learn from real-world use and encounter, as well as its potential, to improve performance. ${ }^{15,16}$

\section{Devices with Artificial Intelligence}

The world of finance was disrupted in 2017 when the first AIpowered exchange traded fund ("ETF") was established. The e-commerce industry has been at the forefront of $\mathrm{AI}$ technology in recent years, employing it to assist consumers in finding products at reduced pricing. ${ }^{14}$ However, healthcare is one of the most important areas where $\mathrm{AI}$ is having an influence, with the market anticipated to exceed $\$ 50$ billion by 2027. Though AI is used throughout the healthcare business, three areas in which it is showing promising results are diagnostics, pathogen tracking, and patient monitoring. ${ }^{14}$

\section{Diagnosis}

The capacity to detect patient problems correctly can have a substantial impact on patient outcomes. Fortunately, new advancements in AI technology are allowing doctors to make a more accurate diagnosis in less time. Deep-learning technology was recently utilised to detect genetic alterations in tumour tissue, a discovery that could lead to less invasive and speedier cancer detection. [14]

\section{Pathogen Tracking}

The COVID-19 shifted our perspective on healthcare. The COVID-19 pandemic demonstrated that the world is far more complex than we could have thought, with notions like "super spreaders" emerging. Traditional virology and public health strategies swiftly proved ineffectual in preparing us for the spread of a fatal virus in our hyperconnected society, forcing us to adopt new approaches. Virologists, healthcare providers, and technology developers were able to collaborate to increase the accuracy of COVID-19 forecasting and mapping models thanks to breakthroughs in $\mathrm{AI}$ networking algorithms.

\section{Patient Monitoring}

The various methods healthcare practitioners are improving patient outcomes by deploying wireless patient monitoring technologies are discussed in relation to remote patient monitoring systems. Healthcare professionals are taking treatment to a whole new level by adding AI into remote patient monitoring systems. One diabetes monitoring startup has unveiled a device that feeds data received from its non-invasive patch into an algorithm that can aid in treatment decision-making. ${ }^{14}$

\section{Challenges Associated with Incorporating Artificial Intelligence in Medical Devices}

$\mathrm{AI}$ is a relatively young issue in the realm of medical devices. Unlike many traditional medical devices, which are often built by teams of material, mechanical, and electrical engineers, AI-based medical devices necessitate knowledge of software coding and programming. ${ }^{15,14}$ Recruiting people with the expertise and skill to construct AI systems is thus one of the most difficult aspects of putting AI into medical devices. Government regulation is another hurdle to overcome when it comes to putting AI into medical equipment. Unlike locating and recruiting AI professionals, which may be simpler for some organizations than others, determining the regulatory framework for AI-based products is a barrier that the entire sector must conquer. ${ }^{14}$

\section{There Appears to Be Three Main Kinds of} Existing AI Medical Device Applications Chronic Disease Management

Healthcare units are employing machine learning to keep an eye on patients via sensors and automate therapy administration via connected mobile apps. ${ }^{17}$ Example: Diabetes and automated insulin delivery.

\section{Medical Imaging}

Businesses are incorporating AI-driven platforms into medical scanning devices to increase image clarity and 
clinical results while lowering radiation exposure. ${ }^{17}$ Example: Computed Tomography scans for liver and kidney abrasions.

\section{Artificial Intelligence and Internet of Things (IoT)}

Companies are combining AI and the Internet of Things (IoT) to better follow up patient adherence to treatment entente and enhance clinical result output. ${ }^{17}$ Example: Philips Healthcare solution for continuous monitoring of patients in critical condition.

\section{Recent Medical Devices with AI}

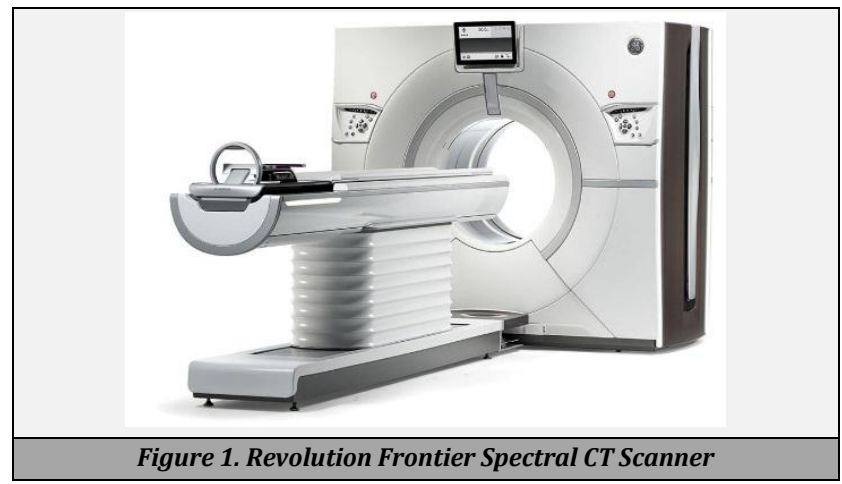

In November 2017, GE Healthcare and NVIDIA declared plans to expand their medical device business. According to reports, the two companies have been working together for ten years to combine NVIDIA's AI platform with GE Healthcare's imaging products.

The researchers claim that artificial intelligence (AI) is being used to increase the speed and accuracy of CT scans. These scans are computer-processed images that combine pictures from several X-rays of the same target. CT scans can produce "cross-sectional images, or slices of the bones, blood arteries, and soft tissues inside [the] body," as opposed to a simple one-dimensional X-ray image. ${ }^{17}$

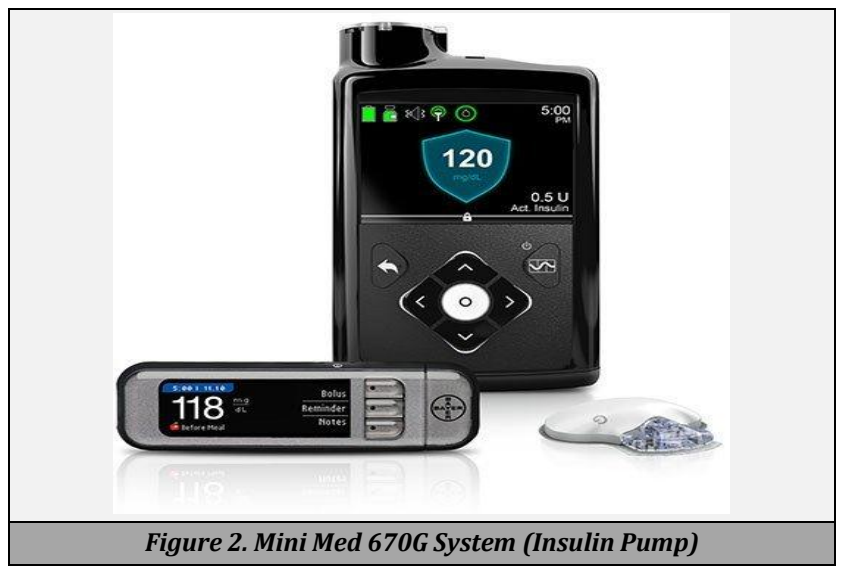

Diabetes is one of the most common lifestyle diseases. A high level of blood glucose i.e. blood sugar, which is a key font of energy from food, causes it. Insulin, a hormone produced by the pancreas, normally aids the fetch of glucose into the body's cells for energy generation. Blood glucose levels can't be managed effectively if insulin isn't present or isn't digested effectively in the body. ${ }^{17}$

Medtronic is one of the medical device firms moving ahead in the AI field, with the goal of assisting diabetics in better managing their condition for a better grade of life. J Evolution Med Dent Sci / eISSN - 2278-4802, pISSN - 2278-4748 / Vol. 10 / Issue 38 / Sept. 20, 2021
Medtronic stated in the year of 2016, Sept that the first users of their Sugar.IQ app, a mobile distinctive assistant developed in conjunction with IBM Watson would be trailed out.

The MiniMed 670G system, an insulin pump authorised by the Food and Drug Administration, was launched in the United States by Medtronic in June 2017. The FDA has approved this technology as the first of its type. The technology, according to the medical device firm, automates the release of a baseline dose of insulin to keep blood sugar stable all over day and night. ${ }^{17}$

\section{Software as a Medical Device}

The scope of software as a medical device is vast. External software ranging from computer-aided detection (CAD) software that performs picture post-processing to help notice breast cancer to software that allows a smart mobile phone to examine images secured from a magnetic resonance imaging (MRI) medical device for diagnostic reasons. ${ }^{15}$

Here are some more examples -

- Other medical gadgets, such as hardware medical devices, and other software as medical device software, as well as a general-purpose software, may be interfaced with software as a medical device. Software that generates settings for a different hardware medical device or another software as a medical device. Software as a medical device, for example, is treatment planning software that provides statistics to a linear accelerator.

- Software as a medical device is software having a medical impetus that runs on a universal purpose computing platform, i.e. a computer platform that has an insufficient medical purpose. Software as a medical device, for example, is software that is designed to diagnose an issue utilising the tri-axial accelerometer on the lodged processor of a consumer camera.

- Software as a medical device is a software that is attached to a hardware medical device but is not required by the hardware medical device to perform its deliberated medical function. It is not an adjunct to the hardware medical device.

Software as a medical device (SaMD) can run on general resolution computing manifestos. A hardware medical device could have software that is used as a medical device and runs on these general motive computing systems. ${ }^{15}$

\section{CONCLUSIONS}

More people will utilise medical devices for health care as medical expenditures continue to rise, especially as more gadgets are developed with lay users in mind. The healthcare industry's brisk development will lead to an advanced revolution in health-care treatment fields. The medical profession must aim to improve the general public's health while also lowering the expense of delivering health care. Medical gadgets, on the other hand, must be made safer, more accessible and useable, and available to a wider range of individuals to facilitate good health care. Human factors engineering provides concepts and techniques that assist the industry in the development of such devices. The application of artificial intelligence to medical devices aids in the expansion of the medical industry and the development of 
new treatments that have the potential to save millions of lives in the future.

Financial or other competing interests: None.

Disclosure forms provided by the authors are available with the full text of this article at jemds.com.

\section{REFERENCES}

[1] Brolin S. Global Regulatory Requirements for Medical Devices. Med Devices 2008.

[2] Olson S. The role of human factors in home health care: workshop summary. Social Sciences 2010;172:180-1.

[3] Medical devices [cited 2021 Sep 11]. https://www.who.int/health-topics/medicaldevices\#tab=tab_1

[4] More N, Ranglani D, Kapusetti G, et al. Summary of current regulatory perspective of medical devices. Futur Pharm Prod Dev Res 2020: p. 849-879.

[5] Crosson FJ. An overview of the medical device industry. In: Report to Congress: Medicare and the Health Care Delivery System. 2017: p. 207-242. http://www.medpac.gov/docs/defaultsource/reports/jun17_ch7.pdf?sfvrsn=0--

[6] Medical Device Industry In India. Investment in Medical Sector [cited $2021 \quad$ Sep 11$]$. https://www.investindia.gov.in/sector/medical-devices

[7] U.S. Bureau of Labour Statistics. Manufacturing in India. 2012. http://www.bls.gov/fls/india.htm
[8] The Development of Medical Devices - technological innovation: comparing development of drugs, devices and procedures in medicine - NCBI Bookshelf [cited 2021 Sep 11]. https://www.ncbi.nlm.nih.gov/books/NBK222708/

[9] What is the Importance of a Medical Device Certificate in India [cited $2021 \quad$ Sep 11$]$. https://corpbiz.io/learning/importance-of-a-medicaldevice-certificate-in-india/

[10] Why is the medical device industry so important? [cited 2021 Sep 11]. http://news.hyperec.com/post/why-isthe-medical-device-industry-so-important

[11] Medical Device Design \& Development [cited 2021 Sep 11]. https://www.meddeviceonline.com/resource/medicaldevice-design-development

[12] Artificial intelligence and machine learning in software as a medical device. FDA [cited 2021 Sep 11]. https://www.fda.gov/medical-devices/softwaremedical-device-samd/artificial-intelligence-andmachine-learning-software-medical-device

[13] Artificial Intelligence in Medical Devices: The Future ATL Technology [cited $2021 \quad$ Sep 11]. https://atltechnology.com/blog/artificial-intelligencein-medical/

[14] Press releases [cited 2021 Sep 11]. https://www.siemens-healthineers.com/press/releases

[15]AI in Medical Devices - Three Emerging Industry Applications. Emerj [cited 2021 Sep 11]. https://emerj.com/ai-sector-overviews/ai-medicaldevices-three-emerging-industry-applications/ 